\title{
Safety of immediate use of totally implantable venous access ports in adult patients with cancer: a retrospective single-center study
}

\author{
Jisu Lee, Sung Mo Hur, Zisun Kim, Cheol Wan Lim \\ Department of Surgery, Soonchunhyang University Bucheon Hospital, Soonchunhyang University College of Medicine, Bucheon, Korea
}

Purpose: Totally implantable venous access ports (TIVAPs) can be used long-term for safe administration of intravenous drugs. TIVAP complications include catheter-related infections, venous thrombosis, extravasation, TIVAP migration, and pain. The relationship between the timing of the first chemotherapy administration after port implantation and complications is controversial. This study aimed to investigate the safety of immediate use of TIVAPs and the associated risk factors for complications.

Methods: Between January 2016 and December 2018, 305 patients (median age, 53 years; 256 women) who underwent TIVAP placement at our institution were included. Chemotherapy was administered within 2 days of implantation. A retrospective analysis of patients' clinical data was performed to investigate catheter days and complications of TIVAPs.

Results: Overall, 305 patients were evaluated over 57,324 catheter days (median, 168 catheter days; interquartile range, 105). The median interval between placement and first use of TIVAPs was 0.98 days. The overall morbidity rate was $2.95 \%$. Nine complications occurred in nine patients, including TIVAP-related infection (4), pain (2), port occlusion (1), thrombosis (1), and scar disunion (1), of which five required port removal (1.64\%). The median number of catheter days before complications occurred was 61 (range, 10-457 days; interquartile range, 51). No complications occurred within 7 days of implantation. Body mass index was an independent risk factor for TIVAP-related complications in the Cox proportional hazards model (multivariable analysis: hazard ratio, $1.221 ; 95 \%$ confidence interval, $1.054-1.414 ; \mathrm{P}=0.008$ ).

Conclusion: This study suggests the safe long-term use of TIVAPs following their immediate chemotherapy administration within 2 days of implantation.

Keywords: Vascular access devices, Cancer chemotherapy agent, Implantable catheters, Catheter-related infections

\section{INTRODUCTION}

Since their first use in the early 1980s, totally implantable venous access ports (TIVAPs) have been safely used in patients with can-

Received: Oct 18, 2018 Accepted: Dec 8, 2021

Correspondence to: Sung Mo Hur

Section of Breast Surgery, Department of Surgery, Soonchunhyang

University Bucheon Hospital, Bucheon, 170 Jomaru-ro, Wonmi-gu,

Bucheon 14584, Korea

Tel: +82-32-621-6267, Fax: +82-32-621-6950

E-mail: smkine@schmc.ac.kr

ORCID: Jisu Lee (https://orcid.org/0000-0003-4971-5069), Sung Mo Hur (https:// orcid.org/0000-0001-8547-5635), Zisun Kim (https://orcid.org/0000-0002-14132800), Cheol Wan Lim (https://orcid.org/0000-0001-9490-4964)

Copyright ( $\odot 2021$ Korean Society of Surgical Oncology

This is an Open Access article distributed under the terms of the Creative Commons Attribution Non-Commercial License (http://creativecommons.org/licenses/by-nc/4.o) which permits unrestricted non-commercial use, distribution, and reproduction in any medium, provided the original work is properly cited. cers to administer long-term chemotherapy [1,2]. TIVAPs can reduce patient discomfort and venous toxicity compared to peripheral vein access. As they are widely used, various complications such as catheter-related infection, venous thrombosis, occlusion, rupture, extravasation, catheter occlusion, pain, and skin disorders have emerged [3].

Several studies have reported the relationship between the timing of the first chemotherapy administration after port insertion and complications [4-6]. However, to date, definite international guidelines for reducing complications due to timing of the first chemotherapy administration after TIVAP insertion have not yet been established. Due to its increased use in clinical practice, TIVAP is also known as chemoport. In our institution, chemoports are used within 2 days of their implantation. This study was performed to investigate the safety of immediate use of TIVAPs and the risk factors for complications. 


\section{METHODS}

\section{Study population and study design}

Between January 2016 and December 2018, 305 patients (median age, 53 years; interquartile range [IQR], 13; 256 women) who underwent insertion of TIVAPs for adjuvant or neoadjuvant chemotherapy in Soonchunhyang University Bucheon Hospital, South Korea were included. A retrospective analysis was performed on the clinical data of the patients to investigate patient characteristics, catheter days, and complications of TIVAPs. We conducted this study in compliance with the principles of the Declaration of Helsinki. The study's protocol was reviewed and approved by the Institutional Review Board of Soonchunhyang University Bucheon Hospital (IRB No. 2021-07-033-001). Informed consent was waived.

\section{Implantation and use of the TIVAPs}

All TIVAPs were implanted by an experienced general surgeon at our institution. The implantations were performed in an operating room using local anesthesia and ultrasound-guided internal jugular vein puncture. Only polyurethane chemoports were used. Heparin was administered to prevent intraluminal thrombosis and the operation was terminated after checking the patency of the port by drawing blood and flushing with saline. After implantation, chest radiography was performed to confirm the appropriate position of the chemoport. No prophylactic antibiotics were administered. All patients received chemotherapy at our institution. The surgical wound was removed in 7 days after implantation, except in cases of wound-related complications.

Table 1. Clinical characteristics of the patients

\begin{tabular}{|c|c|c|c|c|}
\hline Characteristics & $\begin{array}{l}\text { All patients } \\
(n=305)\end{array}$ & $\begin{array}{l}\text { No complications } \\
\qquad(n=296)\end{array}$ & $\begin{array}{l}\text { Complications } \\
\qquad(n=9)\end{array}$ & P-value \\
\hline Age (yr), median (interquartile range) & $53.0(13)$ & $53.2(13)$ & $55.4(14)$ & 0.491 \\
\hline Sex & & & & 0.183 \\
\hline Male & $49(16.1)$ & 49 (16.6) & 0 & \\
\hline Female & $256(83.9)$ & $247(83.4)$ & $9(100)$ & \\
\hline Body mass index $\left(\mathrm{kg} / \mathrm{m}^{2}\right)$, median (interquartile range) & $23.7(4.8)$ & $23.6(4.8)$ & $29.6(6.2)$ & 0.008 \\
\hline Tumor sites & & & & 0.295 \\
\hline Breast & $225(73.8)$ & $217(73.3)$ & $8(88.9)$ & \\
\hline Gastrointestinal tract & $74(24.3)$ & $73(24.7)$ & $1(11.1)$ & \\
\hline Other tumors & $6(1.9)$ & $6(2.0)$ & 0 & \\
\hline Indication for chemotherapy & & & & 0.879 \\
\hline Adjuvant chemotherapy & $266(87.2)$ & $258(87.2)$ & $8(88.9)$ & \\
\hline Neoadjuvant chemotherapy & $39(12.8)$ & $38(12.8)$ & $1(11.1)$ & \\
\hline Anticoagulant therapy & $31(10.2)$ & $28(9.5)$ & $3(33.3)$ & 0.020 \\
\hline Antiplatelets & $27(8.9)$ & $25(8.5)$ & $2(22.2)$ & \\
\hline New oral anticoagulants & $4(1.3)$ & $3(1.0)$ & $1(11.1)$ & \\
\hline
\end{tabular}

The lifespans of TIVAPs, as catheter days, were defined as the number of days from the day of implantation to the day when the catheter was removed or the last follow-up date, whichever came first. The interval days were defined as the number of days from the day of implantation to the day when the port was first used for chemotherapy.

\section{Complications}

TIVAP-related complications in this study included catheter-related infections, pain, thrombosis, and occlusion, and etc. The definitions for each are as follows.

TIVAP-related infection was defined according to the Infectious Diseases Society of America guidelines [7] as one of the following conditions: (1) TIVAP-related bloodstream infection, defined as a positive culture of the catheter tip or reservoir's port of TIVAP associated with a positive peripheral blood culture with the same microorganism (same species and same antibiotic susceptibility testing) or the difference in time to positivity of a blood culture drawn from the catheter versus that from a peripheral vein (positivity of the catheter blood sample was at least 2 hours before that of the peripheral blood sample); (2) Local or general signs of infection, such as fever (temperature $\geq 38.3^{\circ} \mathrm{C}$ or $<36^{\circ} \mathrm{C}$ ) and chills, positive culture of TIVAP (catheter tip or the reservoir's port), and regression of clinical signs of infection after TIVAP removal, despite a negative peripheral blood culture.

Pain as a complication was defined as follows: (1) chronic and persistent moderate to severe pain; (2) pain that occurs during intravenous infusion via chemoport; thus, the chemotherapeutic agent was administered via peripheral intravenous line; or (3)

Values are presented as number (\%) unless otherwise indicated. 
acute pain that occurred from the time of port insertion to the first postoperative day with a score of 4 points on the numeral rating scale (NRS) was excluded.

Catheter-related thrombosis was diagnosed using imaging tests, such as neck ultrasonography or computed tomography (CT).

Catheter occlusion was defined as failure of catheter regurgitation and saline flushing which was caused by adherent collagen and fibrin covering the tip of the catheter and not by catheter malposition or thrombosis.

Complications were defined as early and late complications when they occurred within 7 days of implantation and 7 days after the procedure, respectively.

\section{Statistical analysis}

Continuous data were presented as medians (range, IQR) and categorical data were presented as numbers (proportions). The Cox proportional hazards model was used to assess the influence of each variable on the TIVAP-related complication rate. All statistical analyses were performed using SPSS Statistics version 26.0 (IBM Corp., Armonk, NY, USA). A two-tailed P-value $<0.05$ was considered statistically significant.

\section{RESULTS}

\section{Patient characteristics}

Overall, 305 patients were evaluated over 57,324 catheter days (median catheter days, 168 days; IQR, 105). The median interval between insertion and first use of the TIVAPs was 0.98 days. The characteristics of the study population are summarized in Table 1. The median age of the study population was 53 years (range, 2582 years; IQR,13), of which majority were women ( $n=256,83.9 \%$ ). Thirty-one patients (10.2\%) received anticoagulant therapy with a medical history of hypertension or angina. Among them, 27 patients $(8.85 \%)$ were given antiplatelets and four $(1.3 \%)$ were given new oral anticoagulants. The most common primary site of tumors was the breast $(\mathrm{n}=225,73.8 \%)$ followed by the gastrointestinal tract $(\mathrm{n}=74,24.3 \%)$. As for the purpose of chemoport insertion, 266 patients $(87.21 \%)$ and 39 patients $(12.79 \%)$ received TIVAPs for adjuvant and neoadjuvant chemotherapy, respectively.

\section{Complications}

TIVAP-related complications and clinical characteristics of patients in this study are described in Table 2. Nine patients (2.95\%) had nine complications, which included four TIVAP-related infections, two cases of pain, one port occlusion, one thrombosis, and one scar disunion. Port removal was required in five patients due to catheter-related complications. The median number of catheter

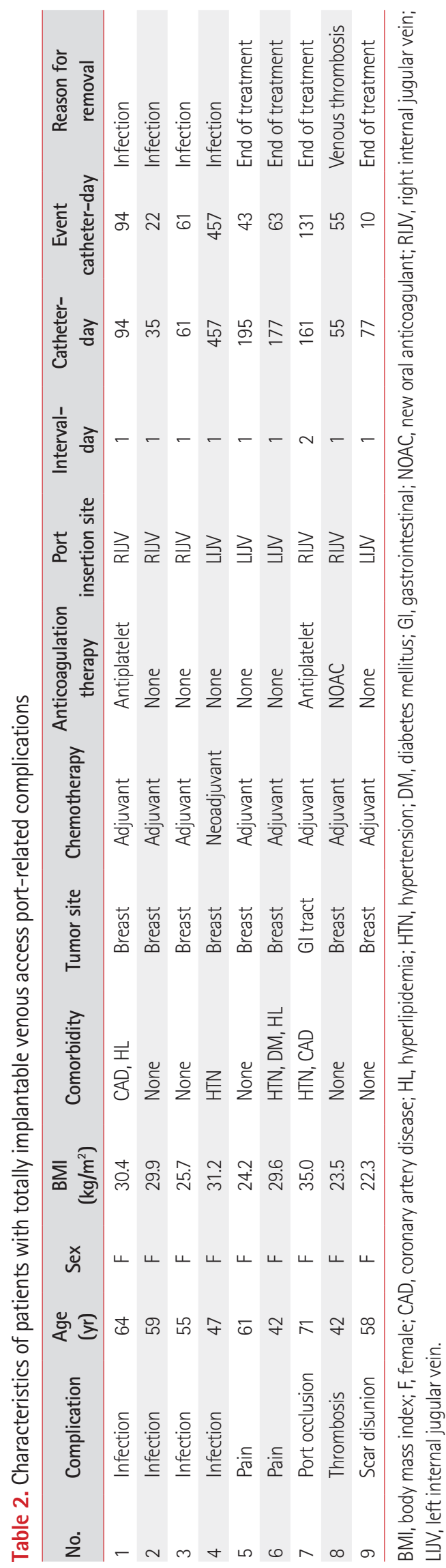


Table 3. Results of univariable and multivariable Cox regression analyses

\begin{tabular}{|c|c|c|c|c|c|c|}
\hline \multirow{2}{*}{ Factor } & \multicolumn{3}{|c|}{ Univariable analysis } & \multicolumn{3}{|c|}{ Multivariable analysis } \\
\hline & $\mathrm{HR}$ & $95 \% \mathrm{Cl}$ & P-value & $\mathrm{HR}$ & $95 \% \mathrm{Cl}$ & P-value \\
\hline Age & 1.023 & $0.958-1.092$ & 0.500 & & & \\
\hline BMI & 1.234 & $1.070-1.423$ & 0.004 & 1.221 & $1.054-1.414$ & 0.008 \\
\hline Hypertension & 1.265 & $0.316-5.057$ & 0.740 & & & \\
\hline Diabetes mellitus & 0.903 & $0.113-7.218$ & 0.923 & & & \\
\hline Coronary artery disease & 0.076 & $0.016-0.364$ & 0.001 & & & \\
\hline Hyperlipidemia & 3.239 & $0.672-15.604$ & 0.143 & & & \\
\hline Right side & 0.362 & $0.097-1.350$ & 0.130 & & & \\
\hline Breast primary & 3.193 & $0.398-25.619$ & 0.275 & & & \\
\hline Anticoagulation therapy & 0.214 & $0.054-0.857$ & 0.029 & 0.284 & $0.070-1.146$ & 0.077 \\
\hline Hemoglobin & 1.081 & $0.604-1.934$ & 0.793 & & & \\
\hline WBC & 1.060 & $0.729-1.543$ & 0.760 & & & \\
\hline
\end{tabular}

$\mathrm{HR}$, hazard ratio; $\mathrm{Cl}$, confidence interval; $\mathrm{BMI}$, body mass index; $\mathrm{WBC}$, white blood cell.

days for complications to occur was 61 (range, 10-457; IQR, 51). No complications occurred within 7 days of TIVAP implantation.

TIVAP-related infections occurred in four patients, due to which the chemoports had to be removed in all four patients. Bacteria identified in the blood culture were Escherichia coli, Klebsiella pneumoniae, and Enterococcus faecalis in each of the three patients, respectively. After sensitivity testing, the infections were controlled with antibiotics following the removal of the chemoports. In the other patient, no bacteria were detected in the blood culture, and the infection symptoms improved after port removal.

TIVAP-related pain was reported in two patients. Both patients complained of chronic pain with an NRS score of 5 for at least 1 month after TIVAP insertion. Chemotherapy was continued as planned for these patients because no abnormal findings were noted on radiography and the chemoports were functional. The pain was managed with intravenous and oral analgesics.

Port occlusion occurred on day 131 after chemoport insertion in one patient. Hence, chemotherapy was performed through the peripheral line and the port was removed after completion of the scheduled chemotherapy.

Thrombosis was reported in one patient. A 42-year-old woman with no specific medical history complained of neck pain and shoulder swelling 55 days after chemoport insertion. Ultrasound imaging of the neck revealed thrombotic obstruction of the right internal jugular vein. The chemoport was removed and oral rivaroxaban $(20 \mathrm{mg})$ was administered once daily for 6 months. A follow-up neck CT scan performed after treatment showed no remaining thrombus.

In one patient with scar disunion, the wound was opened 10 days after implantation due to skin necrosis at the chemoport insertion site; thus, wound revision was performed. Subsequently, the port continued to be used without any further complications.

\section{Risk factors for complications}

The results of the univariate and multivariate Cox regression models are summarized in Table 3. No association was observed between catheter-related complications and patient age, primary tumor site, or port insertion site. The body mass index (BMI) of the patients and catheter-related complications were positively correlated in univariable (hazard ratio [HR], 1.234; 95\% confidence interval $[\mathrm{CI}], 1.070-1.423 ; \mathrm{P}=0.004)$ and multivariable $(\mathrm{HR}$, 1.221; 95\% CI, 1.054-1.414; $\mathrm{P}=0.008$ ) analyses. In univariable analysis, there was a negative correlation between patient's history of anticoagulant therapy and the incidence of complications (HR, 0.214; 95\% CI, 0.054-0.857; $\mathrm{P}=0.029)$, but not in multivariable analysis (HR, 0.284; 95\% CI, 0.070-1.146; $\mathrm{P}=0.077$ ).

\section{DISCUSSION}

This study suggests the safe long-term use of TIVAPs following their immediate chemotherapy administration within 2 days of implantation. Administration of chemotherapy drugs through the peripheral vein begun in the early 1980s and have been reported to cause various complications, such as vascular toxicity, extravasation, and irritability. TIVAPs have been used for chemotherapy worldwide more recently [8] and have been proven safe for longterm use. As the number of patients using TIVAPs increases, understanding the characteristics of its complications has become more important. Several studies have reported the risk factors for TIVAP complications; however, evidence regarding the ideal timing of the first chemotherapy administration after port implantation to reduce complications is limited. 
We carried out a retrospective, single-center study that included 305 TIVAPs over 57,324 catheter days. The overall incidence of complications was $2.95 \%$, which is significantly lower than that reported by Narducci et al. (16.1\%) [5], Zhou et al. (13.2\%) [9], and Hoareau-Gruchet et al. (7.4\%) [10]. This result suggests that even immediate use of chemoports after implantation does not increase the complication rate, making it safe for long-term use. The relationship between the timing of the first chemotherapy administration after port insertion and complications has also been studied in several studies. According to Narducci et al. [5], an interval of at least 8 days may be advisable to reduce complications. They reported that the complication rate was $24.4 \%$ when the interval was $0-3$ days, $17.1 \%$ when it was $4-7$ days, and $12.1 \%$ when it exceeded 7 days. They mainly used the external jugular vein or the cephalic vein for catheterization via the cutdown technique. However, in our study, we catheterized the internal jugular vein using the ultrasonography-guided modified Seldinger technique as less invasive techniques may improve wound healing and chemoport maturation. Similar to our findings, the study of Ozdemir et al. [6] reported that immediate chemotherapy administration after TIVAP implantation is safe in an inpatient setting. Their study involved 180 patients who were initiated on chemotherapy on the day of chemoport implantation, which was mainly performed through the internal jugular vein under ultrasonography guidance. They reported that the acute complication rate was $33.4 \%$, but two-thirds (22.8\%) were minor cutaneous bleeding with no major acute complications. Chronic complication rate was $11.7 \%$ and the symptomatic thrombosis was the most frequent complication (6.1\%) followed by sepsis (1.1\%). In our study, the overall complication rate of $2.95 \%$, which suggests the safety of immediate use of TIVAPs.

In our institution, the first administration of chemotherapy is performed 1 day after TIVAP implantation after confirming the function of the port. This protocol reduces the patient's discomfort in having to visit the hospital for a second time to receive chemotherapy. In addition, as patients are admitted as inpatients after the procedure, postoperative complications such as hematoma, pneumothorax, or hemothorax can be detected and treated in the hospital more immediately and efficiently. Lastly, immediate administration of chemotherapy following TIVAP implantation reduces the patient's hospital stay.

In our study, nine patients had TIVAP-related complications, of whom five needed removal of the chemoports. Among the complications, TIVAP-related infection and pain were the most common, followed by occlusion, thrombosis, and scar disunion; however, there were no major acute complications, such as hemothorax, pneumothorax, or acute thrombosis. Infection is one of the most frequent TIVAP-related complications. According to recent studies, infection rates range from 0.018 events $/ 1,000$ catheter days to 0.35 events/1,000 catheter days in adult cancer patients [11-13]. Various microorganisms, such as bacteria and fungi, are responsible for TIVAP-related infections. Among bacteria, coagulase-negative staphylococci (CoNS) was the most common cause of infection, followed by Staphylococcus aureus, and Candida species are the most frequent among fungi [14]. Some studies have also reported a high incidence of gram-negative bacteria as responsible for TIVAP-related infection, like Klebsiella species and Escherichia coli or Pseudomonas aeruginosa $[13,15,16]$. In our study, bacteria were detected in each of three of the four patients with TIVAP-related infection, and the bacteria were Escherichia coli, Klebsiella pneumoniae, and Enterococcus faecalis, respectively. As CoNS are normal skin flora, infection appears to occur due to insufficient disinfection before port implantation [14]. In our institution, TIVAP implantation is performed under sterile conditions in the operation room, and povidone iodine is used for skin disinfection.

Thrombosis has been the second major long-term complication of catheter use in cancer patients after infection [17]. Recent studies have reported the incidence of thrombosis rates between 2.1\% and $12.8 \%$ [18-20]. Symptoms of thrombosis include difficulty in aspirating or infusing through the catheter, and pain or a burning sensation during drug administration. Other common signs are redness, swelling, and edema [21]. The Doppler ultrasound has been commonly used to confirm the diagnosis of catheter-induced thrombosis because of its high sensitivity (78\% to $100 \%$ ) and specificity ( $82 \%$ to $100 \%)$ as reported in prospective study [22]. In patients with suspected thrombosis and a normal ultrasonography, alternative imaging study like CT should be carried out [23]. When thrombosis is diagnosed, anticoagulation therapy is treatment of choice, and even TIVAP can be removed if necessary [24]. In this study, one case of TIVAP-related thrombosis was reported. The diagnosis was made by ultrasonography. After port removal, anticoagulation therapy with oral rivaroxaban was performed for 6 months, and there was no thrombosis on follow-up CT.

In this study, when analyzing the risk factors for complications, there was no difference in the incidence of complications based on age, primary tumor site, and port insertion location. Whereas, patients with TIVAP-related complications had higher BMI. Obesity has been reported to be associated with TIVAP-related complications in other studies $[20,25,26]$. Ignatov et al. [20] reported that patients with a BMI $>28.75 \mathrm{~kg} / \mathrm{m}^{2}$ had an increased risk of TIVAPrelated complications. In our study, it is worth noting that the complication rate decreased in patients who were previously diagnosed with coronary artery disease. Although the mechanism of this is not clear, a plausible explanation is that anticoagulation therapy for 
coronary artery disease inhibits thrombosis, thereby lowering the incidence of complications of TIVAPs.

This study has several limitations. First, most participants had breast cancer and were receiving adjuvant chemotherapy. The low overall complication rate reported in our study may be due to the lower incidence of infectious complications in solid tumors compared to hematologic tumors and the predominance of young to middle-aged women with no underlying disease in our study population [27]. Second, it was difficult to derive statistically significant results because of the low incidence of complications. Lastly, the immediate use of TIVAPs is protocolized in our institution; hence, analysis based on different interval periods between chemoport implantation and first use of chemoport was not possible.

In conclusion, our results show that the immediate administration of chemotherapy following TIVAP implantation is potentially safe for long-term use and may not increase complications.

\section{CONFLICT OF INTEREST}

No potential conflict of interest relevant to this article was reported.

\section{ACKNOWLEDGMENTS}

This research was supported by Soonchunhyang University Research.

\section{REFERENCES}

1. Voog E, Campion L, du Rusquec P, Bourgeois H, Domont J, Denis F, et al. Totally implantable venous access ports: a prospective longterm study of early and late complications in adult patients with cancer. Support Care Cancer 2018;26:81-9.

2. Vescia S, Baumgartner AK, Jacobs VR, Kiechle-Bahat M, Rody A, Loibl S, et al. Management of venous port systems in oncology: a review of current evidence. Ann Oncol 2008;19:9-15.

3. Kurul S, Saip P, Aydin T. Totally implantable venous-access ports: local problems and extravasation injury. Lancet Oncol 2002;3:68492.

4. Kakkos A, Bresson L, Hudry D, Cousin S, Lervat C, Bogart E, et al. Complication-related removal of totally implantable venous access port systems: does the interval between placement and first use and the neutropenia-inducing potential of chemotherapy regimens influence their incidence? A four-year prospective study of 4045 patients. Eur J Surg Oncol 2017;43:689-95.

5. Narducci F, Jean-Laurent M, Boulanger L, El Bedoui S, Mallet Y, Houpeau JL, et al. Totally implantable venous access port systems and risk factors for complications: a one-year prospective study in a cancer centre. Eur J Surg Oncol 2011;37:913-8.

6. Ozdemir NY, Abali H, Oksuzoglu B, Budakoglu B, Akmangit I, Zengin N. It appears to be safe to start chemotherapy on the day of implantation through subcutaneous venous port catheters in inpatient setting. Support Care Cancer 2009;17:399-403.

7. Mermel LA, Allon M, Bouza E, Craven DE, Flynn P, O'Grady NP, et al. Clinical practice guidelines for the diagnosis and management of intravascular catheter-related infection: 2009 update by the Infectious Diseases Society of America. Clin Infect Dis 2009; 49:1-45.

8. Niederhuber JE, Ensminger W, Gyves JW, Liepman M, Doan K, Cozzi E. Totally implanted venous and arterial access system to replace external catheters in cancer treatment. Surgery 1982;92:70612

9. Zhou J, Qian S, He W, Han G, Li H, Luo R. Implanting totally implantable venous access port via the internal jugular vein guided by ultrasonography is feasible and safe in patients with breast cancer. World J Surg Oncol 2014;12:378.

10. Hoareau-Gruchet F, Rtail R, Sulaj H, Khirnetkina A, Reyt E, Righini CA. Complications after insertion of a totally implantable venous access port in patients treated with chemotherapy for head and neck squamous cell carcinoma. Ann Otolaryngol Chir Cervicofac 2009; 126:43-52.

11. Shim J, Seo TS, Song MG, Cha IH, Kim JS, Choi CW, et al. Incidence and risk factors of infectious complications related to implantable venous-access ports. Korean J Radiol 2014;15:494-500.

12. Busch JD, Herrmann J, Heller F, Derlin T, Koops A, Adam G, et al. Follow-up of radiologically totally implanted central venous access ports of the upper arm: long-term complications in 127,750 catheter-days. AJR Am J Roentgenol 2012;199:447-52.

13. Wang TY, Lee KD, Chen PT, Chen MC, Chen YY, Huang CE, et al. Incidence and risk factors for central venous access port-related infection in Chinese cancer patients. J Formos Med Assoc 2015;114:1055-60.

14. Pinelli F, Cecero E, Degl'Innocenti D, Selmi V, Giua R, Villa G, et al. Infection of totally implantable venous access devices: a review of the literature. J Vasc Access 2018;19:230-42.

15. Hsu JF, Chang HL, Tsai MJ, Tsai YM, Lee YL, Chen PH, et al. Port type is a possible risk factor for implantable venous access port-related bloodstream infections and no sign of local infection predicts the growth of gram-negative bacilli. World J Surg Oncol 2015;13: 288.

16. Chang L, Tsai IS, Huang SI, Shih CC. Evaluation of infectious complications of the implantable venous access system in a general oncologic population. Am J Infect Control 2003;31:34-9.

17. Teichgraber U, Nagel SN, Kausche S. Evaluation of correlations between underlying disease and port complications. Rofo 2014;186: 
496-500.

18. Piran S, Ngo V, McDiarmid S, Le Gal G, Petrcich W, Carrier M. Incidence and risk factors of symptomatic venous thromboembolism related to implanted ports in cancer patients. Thromb Res 2014;133:30-3.

19. Biffi R, Orsi F, Pozzi S, Pace U, Bonomo G, Monfardini L, et al. Best choice of central venous insertion site for the prevention of catheter-related complications in adult patients who need cancer therapy: a randomized trial. Ann Oncol 2009;20:935-40.

20. Ignatov A, Hoffman O, Smith B, Fahlke J, Peters B, Bischoff J, et al. An 11-year retrospective study of totally implanted central venous access ports: complications and patient satisfaction. Eur J Surg Oncol 2009;35:241-6.

21. Sousa B, Furlanetto J, Hutka M, Gouveia P, Wuerstlein R, Mariz JM, et al. Central venous access in oncology: ESMO clinical practice guidelines. Ann Oncol 2015;26 Suppl 5:v152-68.

22. Surov A, Jordan K, Buerke M, Arnold D, John E, Spielmann RP, et al. Port catheter insufficiency: incidence and clinical-radiological correlations. Onkologie 2008;31:455-61.

23. Baskin JL, Pui CH, Reiss U, Wilimas JA, Metzger ML, Ribeiro RC, et al. Management of occlusion and thrombosis associated with long-term indwelling central venous catheters. Lancet 2009;374: 159-69.

24. Debourdeau P, Farge D, Beckers M, Baglin C, Bauersachs RM, Brenner B, et al. International clinical practice guidelines for the treatment and prophylaxis of thrombosis associated with central venous catheters in patients with cancer. J Thromb Haemost 2013; 11:71-80.

25. Lefebvre L, Noyon E, Georgescu D, Proust V, Alexandru C, Leheurteur M, et al. Port catheter versus peripherally inserted central catheter for postoperative chemotherapy in early breast cancer: a retrospective analysis of 448 patients. Support Care Cancer 2016; 24:1397-403.

26. Wyschkon S, Loschmann JP, Scheurig-Munkler C, Nagel S, Hamm B, Elgeti T. Apparent migration of implantable port devices: normal variations in consideration of BMI. J Vasc Access 2016;17:15561.

27. Chen IC, Hsu C, Chen YC, Chien SF, Kao HF, Chang SY, et al. Predictors of bloodstream infection associated with permanently implantable venous port in solid cancer patients. Ann Oncol 2013;24: 463-8. 